\title{
Easy Authoring of Variable Gestural Expressions for a Humanoid Robot
}

\author{
Tamara Flemisch, André Viergutz, Raimund Dachselt \\ Interactive Media Lab \\ Technische Universität Dresden \\ 01062 Dresden, Germany \\ tamara.flemisch@mailbox.tu-dresden.de, (andre.viergutz|raimund.dachselt)@tu-dresden.de
}

\begin{abstract}
This demonstration on the Nao robot uses the selection of behavior based on a feedback type and an expressivity value to generate and execute appropriate feedback. It aims to ease the authoring process by using variable gestures.
\end{abstract}

\section{Categories and Subject Descriptors}

I.2.9 [Artificial Intelligence]: Robotics-Operator Interfaces

\section{Keywords}

Human-robot Interaction; Gesture; Expressivity

\section{VARIABLE GESTURAL EXPRESSIONS}

The selection of gestures and the expressivity of a robot's behaviour are critical factors for suitable and understandable feedback. To provide more variability, the parametrization of gestures is explored by Hartmann et al. [1] who specify parameters such as Spatial Extent or Repetition which increase the expressivity of a gesture. However, in everyday communication people usually want to increase the expressiveness of a certain expression, intention or emotion, for instance, happiness, sadness or suprise. Therefore, we developed a set of variable gestures which can be adjusted by a feedback type and an expressivity value.

In view of communication and inspired by Hartmann et al. 11], we divided the gestures into the feedback types Positive/Acceptance, Negative/Denial, Incomprehension, Waiting/Neutral, Introduction/Greeting and Ending/Goodbye which form classes with own gesture sets. To support variability and potential for increase of a certain feedback category, the global parameter expressivity is subsequently mapped to the underlying parameters for each gesture.

Permission to make digital or hard copies of part or all of this work for personal or classroom use is granted without fee provided that copies are not made or distributed for profit or commercial advantage, and that copies bear this notice and the full citation on the first page. Copyrights for third-party components of this work must be honored. For all other uses, contact the owner/author(s). Copyright is held by the author/owner(s).

HRI'14, March 3-6, 2014, Bielefeld, Germany.

ACM 978-1-4503-2658-2/14/03.

http://dx.doi.org/10.1145/2559636.2559786.

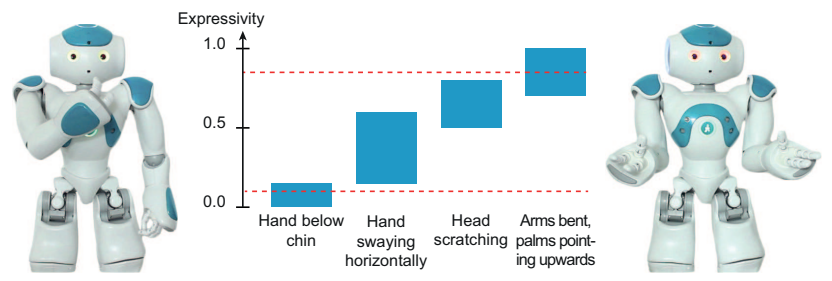

Figure 1: Performed gestures for feedback type Incomprehension with expressivity: 0.1 (left) and 0.9 (right).

\section{IMPLEMENTATION ON THE NAO}

We implemented these variable gestures to use them for communicating with a robot, in particular with the humanoid robot Nao by Aldebaran Robotics. The internal process of choosing a proper gesture from a total of 26 possible basic gestures with over 50 variations happens in two steps. First, the feedback type is evaluated and the appropriate gesture set is selected. Afterwards, the system interprets the value for the expressivity parameter and chooses a gesture from a predefined set of approximately ten variations per category. To evoke a behaviour generated by variable gestures there are two possibilities. On the one hand, the interacting user can talk to Nao which understands particular utterances, like simple statements and questions and reacts on them with a suitable gesture based on predefined values which show the appropriate feedback. On the other hand, the user can specify the parameters manually. These are the aforementioned feedback types and an expressivity which is a value between 0 for almost no expressiveness and 1 for very expressive behaviour. Two Incomprehension gestures are shown in Figure 1 which are created according to the user's input.

As a result, we developed an application and a gesture library which are straightforward and intuitive in usage and enhance the communication with nonverbal cues and convincing feedback.

\section{REFERENCES}

[1] B. Hartmann, M. Mancini, and C. Pelachaud. Implementing expressive gesture synthesis for embodied conversational agents. In GW'05 Proc. of Gesture in Human-Computer Interaction and Simulation, pages 188-199, Berlin, Heidelberg, 2006. Springer-Verlag. 